\title{
Variation between Antagonistic Activity of Rosmarinus officinalis Essential Oil and Biosynthesized Silver Nanoparticles from the Essential Oil against Some Fungi
}

\author{
Rusol M. Al Bahrani, Ali M. Najem* and Ibrahim J. Abed \\ Department of Biology, College of science, University of Baghdad, Baghdad-Al-Jadiria, Iraq. \\ * Corresponding Author: alimuayyed1111@gmail.com
}

\begin{abstract}
This study was conducted to evaluate the antagonistic activity of Rosmarinus officinalis L. (Rosemary) essential oil at different concentrations (500, 1000, 2000 or $3000 \mathrm{ppm}$ ) against three different fungi (Aspergillus niger, Penecillium notatum and Candida sp.), these isolates were isolated from air. On the other hand, a combination between the Rosemary essential oil and synthesized nanoparticles were tested against the isolated fungi. Results revealed that Rosemary essential oil exhibited the highest antifungal potentiality comparing to the antagonisty of the biosynthesized nanoparticles. The concentration $3000 \mathrm{ppm}$ of the essential oil showed 100\% mycelial growth inhibition of all the fungi. Aspergillus niger was the most sensitive which showed no growth in the concentrations 1000, 2000 or $3000 \mathrm{ppm}$. The biosynthesized silver nanoparticles from Rosemary essential oil exhibited a considerable fungicidal activity against all the fungal isolates. The inhibition of all fungal isolates increased as the concentrations of the synthesized silver nanoparticles increased. Asp. niger was the most sensitive fungi to the inhibitory impact of the Rosemary nanoparticles and Penicillium notatum manifested mild response, while Candida sp. was less sensitive. [DOI: 10.22401/ANJS.21.4.09]
\end{abstract}

Keywords: Rosmarinus officionalis, silver nanoparticles, antifungal activity, growth inhibition.

\section{Introduction}

Silver nanoparticles (AgNPs) have been considered as one of the important techniques because of the extensive applications in various fields. (AgNPs) was discovered for over 2000 years, in many areas among nanoparticles, (AgNPs) in particular are known for their versatile applications in consumer goods, food processing industries, textile industries, medical industries and for being an efficient antimicrobial agent [1]. Several methods are mentioned to synthesize AgNPs, which include chemical reduction، electrochemical reduction and LangmuirBlodgett .Many studies have been published dealing with the synthesis of metal nanoparticles using plant extracts [2, 3]. Plants and their extracts have been used since the dawn of civilization, the secondary metabolic compounds naturally produce by plants are taking an inevitable role in as a substitute for the chemical therapy [5]. Nowadays, plant extract has been used as reducing and capping agent for the synthesis of nanoparticles, the essential oil of Rosemary and its chemical compounds, antimicrobial and antifungal impacts have been studied worldwide [4]. $R$. officinalis is a small evergreen perennial shrub, belonging to the Lamiaceae family; its name is derivesed from the Latin for "dew" (ros) and "sea" (marinus), or "dew of the sea". The leaves are used as flavoring and preservatives in the food industries, or for medical or dermal treatment [6]. Food spoilage due to overgrowth of many fungi even in preservative foods, the contaminated fungi produce many toxins and enzymes which cause side reactions affect healthcare of the consumers in addition to the taste and odor of these foods result in decrease their values, furthermore, these reactions create byproducts responsible for many diseases related to intake of the contaminated foods with fungi, thus many studies have been conducted to eliminate this threat [7]. Fungi infect plant in the field and post-harvest even during food industry, these infections are related to many diseases which cause by many hydrolytic enzymes produce by these fungi [8]. Nourishment values of crops decrease due to post harvest infections by molds result in dramatic economical losses [9]. Awareness of people has increased about healthcare and hazard risks associated to commodities processed 
with chemical fungicidal compounds or compounds extracted from natural sources [10]. New line of therapeutically active procedures have been evolved with high antagonistic activity against broad spectrum of pathogens, safety and with low impact on environment, these procedures are depending on extraction, isolation and purification of active compounds from medicinal plants [11].

The aims of this study were to synthesise AgNP by rosemary essential oil and evaluate the synthesized nanoparticals from $R$. officionalis L. (Rosmary) essential oil as antifungal agent against different fungal isolates.

\section{Materials and Methods Plant collection}

Rosmarinus officinalis (Rosemary) leaves were collected midday from the gardens of Baghdad University, Iraq and air dried at room temperature. The plant was identified in the Biology department's Herbarium, College of Science University of Baghdad.

\section{Extraction of volatile oil}

Dried leaves of Rosemary were ground finely and placed in Clevenger apparatus to extract the volatile oil using hydro distillation method by applying a ratio 1:5 between plant and distilled water [12].

\section{Isolation and identification of fungi}

Fungi were collected from air, then cultured on SDA (Sabroud dextrose agar) then incubated for 5-7 days at $25^{\circ} \mathrm{C}$. Persistent laboratory and sterilized attempts were conducted to gain pure fungal isolates. All pure fungal isolates were identified morphologically and under light microscope according to standard keys of fungi classification .

\section{Evaluation of the inhibitory effect of the extracted oil against isolated fungi Experimental design 1}

Sabroud dextrose agar plates were prepared with different concentrations of Rosmary essential oil $(500,1000,2000$ or 3000) $\mathrm{ppm}$ for each isolated fungi by mixing $(50,100,200$ or $300 \mu \mathrm{l})$ subsequently of the volatile oil with equal volume of DMSO
(Dimethyl sulfoxide) dissolved in SDA medium approaching the final volume $100 \mathrm{ml}$ then left to solidify in plates. Eight milimeters of mycelia growth for each fungi was cut off and located in the center of each plate. These plates were incubated for 7 days. Control plate and two replicates were prepared then the anti fungal activity of each concentration of the studied oil was calculated by measuring the Growth inhibition using the following formula:

Growth inhibition\% = [Growth in control Growth in treatment] /Growth in control $\times 100$ [13].

\section{Experimental design 2}

This experiment depended on the agar well diffusion methods including preparation of sterilized potato dextrose agar plates, an inoculum from $18 \mathrm{hrs}$. broth of each fungi was swabbed, $6 \mathrm{~mm}$ wells were made in each plate then $0.1 \mathrm{ml}$ of each concentration of the oil were added. Anti fungal activity of the oil was evaluated by measuring diameters of the inhibition zones [14].

\section{Biosynthesis of silver nanoparticle}

A mixture of $5 \mathrm{ml}$ of Rosmarinus officinalis volatile oil and $45 \mathrm{ml}$ of AgNO3solution was prepared, the prepared solution was kept in a clean and sealed flask placed in sunlight for 3 days.

\section{Properties of nanoparticles}

Solution of AgNO3 and $R$. officinalis volatile oil were prepared, then the change in color indicated the formation of nanoparticales proved with AFM device. Estimation the size of atoms was carried out with spectro device.

\section{Results and Discussion Isolation of fungi}

A. niger, $P$. notatum. and Candida sp. were purely isolated out of many identified fungi and used in this study as shown in Figures (1-3). 


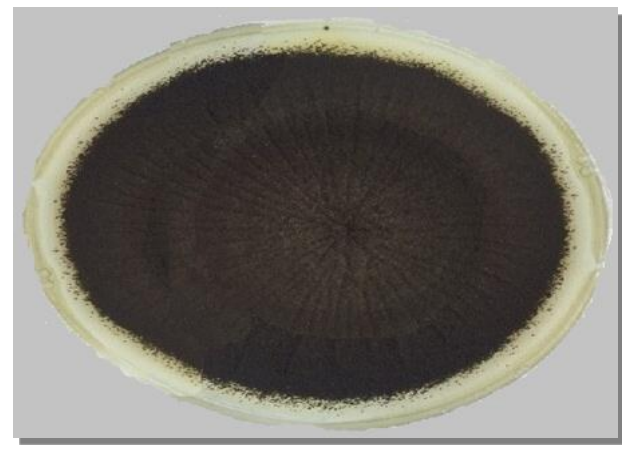

Fig.(1): Aspergillus niger.

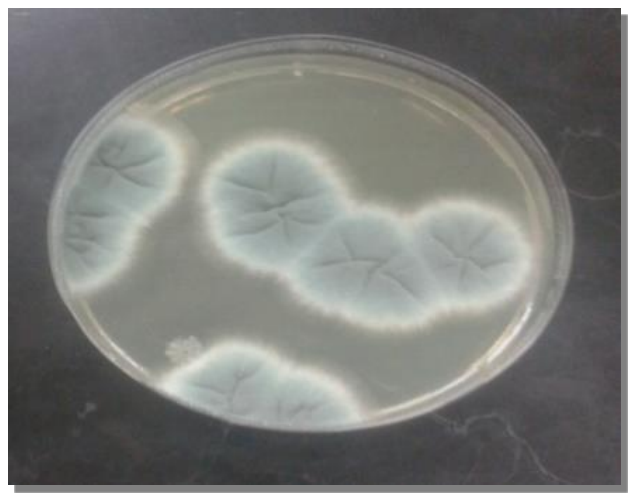

Fig.(2): Penicillium notatum.

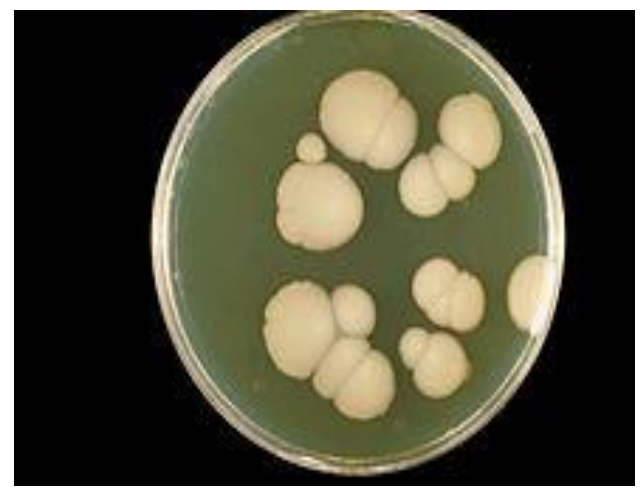

Fig.(3): Candida sp.
Yield of Rosemary volatile oil

The yield of the volatile oil resulted from extraction $2.7 \mathrm{ml}$ of the oil from $150 \mathrm{~g}$ of Rosemary dried leaves was $1.8 \%$ by using Clevenger apparatus.

\section{Fungal growth inhibition of Rosemary volatile oil}

Volatile oil at the concentrations 500, 1000,2000 or $3000 \mathrm{ppm}$ were used to estimate the fungicidal potentiality of Rosemary volatile oil, which manifested remarkable impacts on fungal colony diameters as shown in Table (1), approaching 100\% growth inhibition as appeared in Table (2). The enormous effect of Rosemary oil could be clearly detected on A. niger which caused complete inhibition of the mycelial growth in three out of four concentrations $(3000,2000$ or $1000 \mathrm{ppm})$ comparing to $P$. notatum and Candida sp. Full growth was inhibited only by the highest concentration (3000) ppm indicating the huge effect of Rosemary volatile oil on A. Niger. Table (2) shows that Candida sp. occupied mild range of affection by Rosemary volatile oil, that affection came in a regular pattern, the inhibitory impact of the oil increased as the concentrations increased. $P$. notatum. manifested $100 \%$ growth inhibition at $3000 \mathrm{ppm}$ of the oil followed by a sharp decrease in the fungicidal potentiality at the concentrations 2000, 1000 and $500 \mathrm{ppm}$.

Table (1)

Inhibitory effect of Rosemary volatile oil concentrations (ppm) against three fungi by measuring diameters of fungal colony (mm).

\begin{tabular}{|c|c|c|c|c|}
\hline \multirow{2}{*}{ Fungal isolates } & \multicolumn{4}{|c|}{ Volatile oil (ppm) } \\
\hline & 3000 & 2000 & 1000 & 500 \\
\hline Aspergillus niger & No growth & No growth & No growth & $8.7 \pm 0.45$ \\
\hline Penecillium notatum & No growth & $7.83 \pm 0.26$ & $8.5 \pm 0.43$ & $7.5 \pm 0.45$ \\
\hline Candida sp. & No growth & $2 \pm 0$ & $3 \pm 0.26$ & $4 \pm 0.33$ \\
\hline
\end{tabular}

The determination of the percentage inhibition of diameter growth for all strains have shown that of Rosemary volatile oil concentrations (ppm) exhibited a higher inhibition ability Table (1). 
Table (2)

Percentages of growth inhibition of three fungi resulted from the fungicidal capacity of Rosemary volatile oil concentrations.

\begin{tabular}{|c||c|c|c|}
\hline \multirow{2}{*}{$\begin{array}{c}\text { Oil } \\
\text { Conc. (ppm) }\end{array}$} & \multicolumn{3}{|c|}{ Growth inhibition \% } \\
\cline { 2 - 4 } & Aspergillus niger & Penecillium notatum & Candida sp. \\
\hline \hline 3000 & 100 & 100 & 100 \\
\hline \hline 2000 & 100 & 39.7 & 60 \\
\hline 1000 & 100 & 34.6 & 40 \\
\hline \hline 500 & 87.5 & 42.3 & 20 \\
\hline
\end{tabular}

Fungal growth inhibition induced by Rosemary volatile oil concentrations, it was dependent mostly on the concentration (varying from $3000 \mathrm{ppm}$ to $500 \mathrm{ppm}$ ) and type of fungi.

\section{Biosynthesized silver nanoparticles}

The resulted nanoparticles of Rosemary volatile oil varied in size and shape as shown in Fig.(4). Fig.(5) shows the distinctions in diameter, granularity, cumulating and distribution of silver nanoparticles.

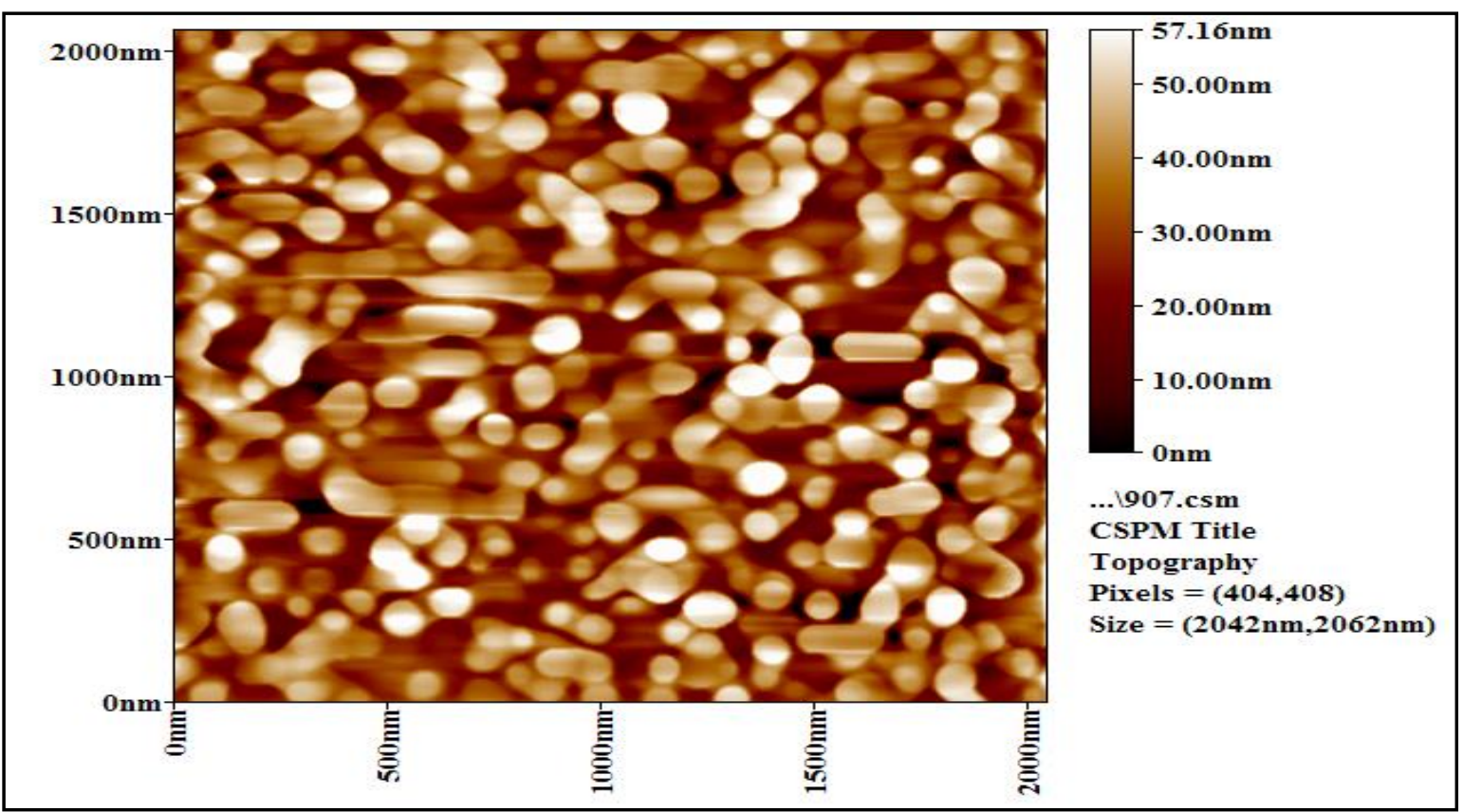

Fig.(4): Characterization of silver nanoparticles as size and shape.

Ag-nano particles might be referred to spherical shape of nanoparticles that contributed of increased surface area then increased activity for nanoparticles. 


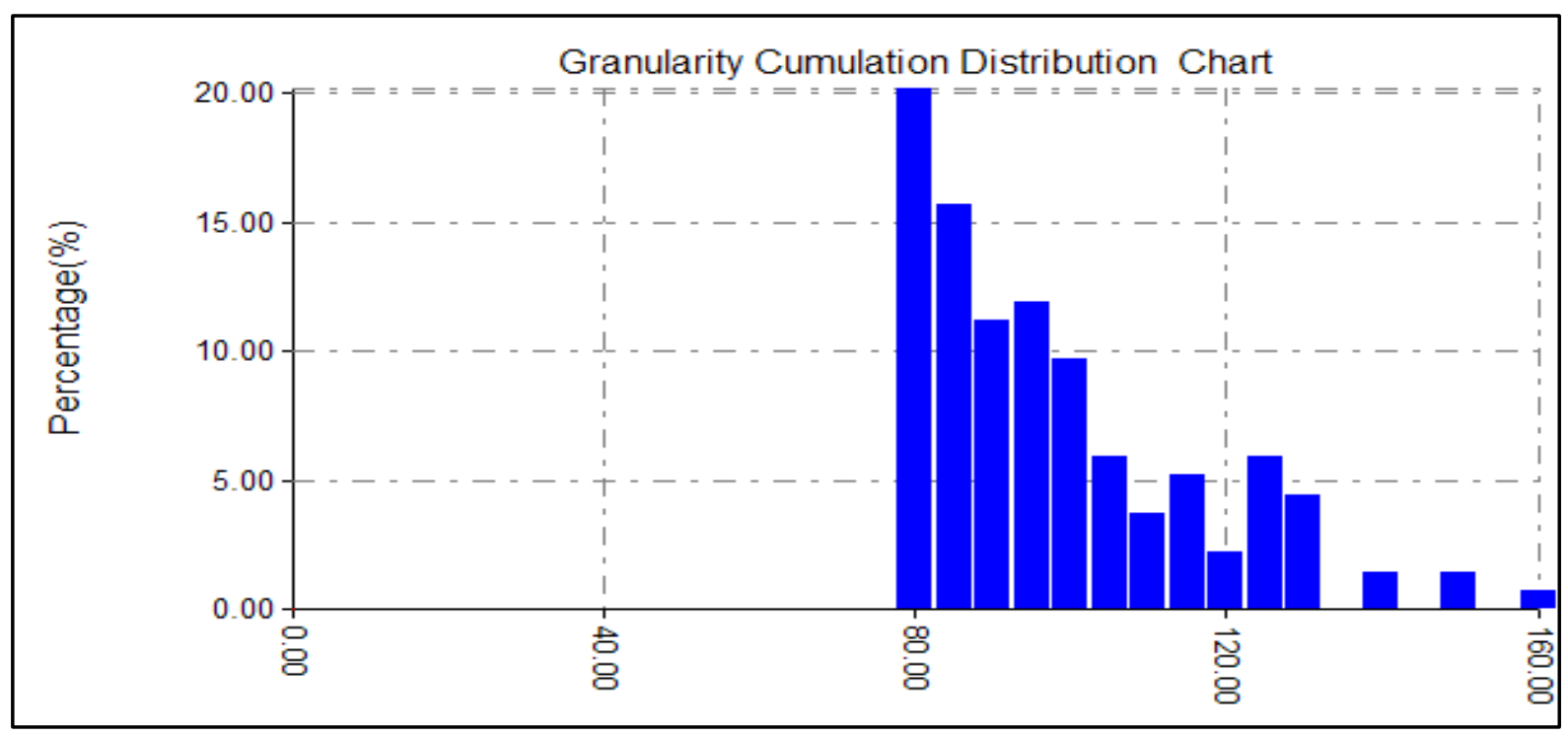

Diameter (nm)

Fig.(5): Average diameter of silver nanoparticles, Granularity, Cumulation and distribution.

The distribution curve for particle size. Intensity, which was obtained from biosynthesis AgNPs reveals that main diameter is $80 \mathrm{~nm}$.

Antifungal activity of synthesized silver nanoparticles

Table (4) shows that the synthesized silver nanoparticles from Rosemary volatile oil have strong antifungal activity depending on resulted diameters of inhibition zones of the fungi included in this study. Basing on the outcome data in Table (4) the inhibition zones of all fungal isolates increased as the concentrations of the synthesized silver nanoparticles from Rosemary volatile oil increased. A. niger was more sensitive to the inhibitory impact of the Rosemary nanoparticles and Candida sp. was the less sensitive, while $P$. notatum Manifested mild response. In a comparison between antifungal potentiality of Rosemary volatile oil in Table (3) and those of silver nanoparticles synthesized from Rosemary volatile oil data and in Table (4) reveals that both have the strongest effects against $A$. niger. Rosemary oil possessed the weakest influence against $P$. notatum, while the synthesized nanoparticles exhibited the weakest effect against Candida sp., mild influence in contrast to the results pre mentioned in the case of weakest impacts.
Table (4)

Estimation of inhibitory effects of synthesized silver nanoparticles against fungal isolates assessed by distinctions in diameters of inhibition zones ( $\mathrm{mm})$.

\begin{tabular}{|c|c|c|}
\hline $\begin{array}{l}\text { Fungal } \\
\text { isolate }\end{array}$ & $\begin{array}{c}\begin{array}{c}\text { Concentration } \\
\text { of silver } \\
\text { nanoparticles \% }\end{array} \\
\end{array}$ & 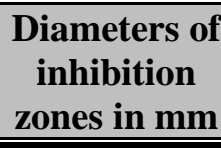 \\
\hline \multirow{4}{*}{$\begin{array}{l}\text { Aspergillus } \\
\quad \text { niger }\end{array}$} & 25 & 16 \\
\hline & 50 & 17 \\
\hline & 75 & 20 \\
\hline & 100 & 23 \\
\hline \multirow{4}{*}{$\begin{array}{l}\text { Penecillium } \\
\text { notatum }\end{array}$} & 25 & 13 \\
\hline & 50 & 14 \\
\hline & 75 & 15 \\
\hline & 100 & 16 \\
\hline \multirow{4}{*}{ Candida sp. } & 25 & 11 \\
\hline & 50 & 13 \\
\hline & 75 & 16 \\
\hline & 100 & 16 \\
\hline
\end{tabular}

\section{Discussion}

Versatile antimicrobial capacity of silver nanoparticles have been encouraged to use it in many matrices and incorporated in various industrial and biomedical utilities [15]. Despite many published studies illustrated the mechanism of action of several chemical components in many volatile oils but the chemical analytical data about most of these components still incomplete. Recent works have illustrated that nanoparticles incorporated 
with volatile oils posses distinct antimicrobial activity against multidrug- resistant microbial agents "due to an increase in chemical stability and solubility, decreased rapid evaporation and minimized degradation of active essential oil components" [16]. Interplay role of essential oils as a broad spectrum anti microbial agents derived from bio viability, high pharmaceutical activity and less toxicity, in addition to their components especially terpenoids which possess a short carbon chain which play an important role in the aid of their high hydrophobicity to bind tightly with lipids in cell membranes of pathogens, which affects tremendously on the permeability of these membranes including mitochondrial membrane which is paving posteriorly to pathogen demise [17,18]. Likewise, another study analyzed the chemical components of seven essential oils and Rosemary was included and the study examined three chemotype of these oils: Cineol, Verbenone and Camphor against Candida albicans. The Cineol was the highest effective against the yeast, the major components in the oil was 1,8 cineole, $\alpha$-pinene and camphor and tested individually, the strongest fungicidal effect was $\alpha$-pinene, but not in camphor or 1,8 cineole [19]. Volatile oils from eight plants including $R$. officinalis were examined for their inhibitory effect against common spoilage fungus $A$. niger, all essential oils showed high antagonistic activity in lowest concentrations except Majorana hortensis (Marjoram) and $R$. officinalis (Rosemary) exhibited strong inhibitory effect at high concentrations [20]. Comparatively, a study was conducted to establish the antifungal activity of Origanum vulgare, $R$. officinalis and Lavandula angustifolia (Lamiaceae) essential oils and biocide benzalkonium chloride against six fungi included $A$. niger and $P$. notatum., $O$. vulgare and biocide benzalkonium chloride manifested the highest funicidal potentiality, while $R$. officinalis and L. angustifolia essential oils showed mild impacts [20].

\section{Conclusion}

Silver nanoparticles were biosynthesized from a combination between $R$. officinalis volatile oil and $\mathrm{AgNO} 3$ solution, this functionalized nanoparticles were scanned to determine the diameter for silver nanoparticles, Granularity, Cumulation and Distribution. Rosemary volatile oil and the biosynthesized silver nanoparticles showed antifungal activity against $A$. niger, $P$. notatum and Candida sp., Rosemary volatile oil exhibited the highest antagonistic effect.

\section{References}

[1] Sharma V., Shukla R. K., Saxena N., Parmar D., Das M., and Dhawan A., "DNA damaging potential of zinc oxide nanoparticles in human epidermal cells", Toxicology Letters, 185, 211-218, 2009.

[2] Farghaly F. A and Nafady N. A., "Green Synthesis of Silver Nanoparticles Using Leaf Extract of Rosmarinus officinalis and Its Effect on Tomato and Wheat Plan", Journal of Agricultural Science; 7 (11), 277-287, 2015.

[3] Dhiman, Kundu J., Kumar V., Kumar R. and Chakarvarti S.K., "Eco-friendly Synthesis and Characterization of Silver Nanoparticles and Evaluation of Their Antibacterial Activity", American Journal of Materials Science and Technology, 3(1), 13-21, 2014.

[4] Jiang H., Manolache S., Wong A.C.L., Denes F.S., "Plasma-enhanced deposition of silver nanoparticles onto polymer and metal surfaces for the generation of antimicrobial characteristics", J. Appl. Polym. Sci., 93, 1411-1422, 2004.

[5] Danida, "Review report on strengthening the medicinal plants resource base in India in the context of primary health care project", 43, 2001.

[6] Mulas M., Francesconi A.D.H., Perinu B. and Del Vais E., "Selection of rosemary (Rosmarinus officinalis L.) cultivars to optimize biomass yield", J. Herbs, Species \& Medicinal Plants 9(2-3), 133-138, 2002.

[7] Becker R.O., "Silver ions in the treatment of local infections", Met. Based Drugs, 6, 311-314, 1999.

[8] Tankhiwale R., Bajpai S.K., "Silvernanoparticle-loaded chitosan lactate films with fair antibacterial properties", J. Appl. Polym. Sci., 115, 1894-1900, 2010.

[9] Duran N., Marcato P.D., Alves O.L., Da Silva J.P.S., De Souza G.I.H., Rodrigues 
F.A., Esposito E., "Ecosystem protectection by effluent bioremediation: silver nanoparticles impregnation in a textile fabric process", J. Nanopart. Res., 12، 285292, 2010.

[10] Chainy G.B.N., "Anethole blocks both early and late cellular responses transduced by tumor necrosis factor: effect on NF- $\mathrm{BB}$, AP-1, JNK, MAPKK and apoptosis", Oncogene, 19, 2943-2950, 2000.

[11] Mulas M., Francesconi A.D.H., Perinu B., and Del Vais E., "Selection of rosemary (Rosmarinus officinalis L.) Cultivars to optimise biomass yield", J. Herbs, Species \& Medicinal Plants, 9 (2-3), 133-138, 2002.

[12] British Pharmacopoeia, Determination of Volatile Oil in Drugs. The Pharmaceutical Press, London, 1963.

[13] Wang S.Y., Wu C., Chu F., Chien S., Kuo Y., Shyur L., and Chang S., "Chemical composition and antifungal activity of essential oil isolated from Chamaecyparis formosensis", Matsum. Wood. Holzforschung, 59, 295-299, 2005

[14] Valgas C., De Souza S.M., Smânia E.F.A., Smânia Jr. A., "Screening methods to determine antibacterial activity of natural products", Braz. J. Microbiol., 38, 369-380, 2007.

[15] Sarkar S., Jana A.D., Samanta S.K.. and Mostafa G., "Facile synthesis of silver nanoparticles with highly efficient antimicrobial property", Polyhedron, 26, 44194426, 2007.

[16] Chouhan S., Sharma K. and Guleria S., "Antimicrobial Activity of Some Essential Oils-Present Status and Future Perspectives", Medicines, 4, 58, 2017.

[17] Solórzano-Santos F. and MirandaNovales M.G., "Essential oils from aromatic herbs as antimicrobial agents", Current Opinion in Biotechnology, 23, 136141, 2012.

[18] Lv F., Liang H., Yuan Q., Li C., "In vitro antimicrobial effects and mechanism of action of selected plant essential oil combinations against four food-related microorganisms", Food Research International, 44, 3057-3064, 2011.

[19] Matsuzaki Y., Tsujisawa T., Nishihara T., Nakamura M. and Kakinoki Y., "Antifungal activity of chemotype essential oils from rosemary against Candida albicans", Open Journal of Stomatology, 3, 176-182, 2013.

[20] Baratta T.M., Dorman D.J. H., Deans G.S., Figueiredo C.A., Barroso G. J. and Ruberto G., "Antimicrobial and antioxidant properties of some commercial essential oils", Flavour and Fragranace Journal, 13, 235-244, 1998. 